Supporting Information for:

\title{
A Peptoid-Based Combinatorial and Computational Approach to Developing Ligands for Uranyl Sequestration from Seawater
}

\author{
Bernard F. Parker ${ }^{\dagger, t}$, Abigail S. Knight $t^{\dagger}$, Sinisa Vukovic ${ }^{\S}$,John Arnold ${ }^{*},+,+$, and Matthew B. \\ Francis $^{*}, \dot{\dagger}, \|$ \\ $\dagger$ Department of Chemistry, University of California, Berkeley, California 94720, United States \\ $\$$ Chemical Sciences Division and "Materials Sciences Division, Lawrence Berkeley National \\ Laboratory, Berkeley, California 94720, United States \\ $\S$ Chemical Sciences Division, Oak Ridge National Laboratory, Oak Ridge, Tennessee 37831, \\ United States
}

\section{Peptoid synthesis}

\section{General procedures and methods}

Peptoids were synthesized according to procedures in our previous work. ${ }^{1}$

Tentagel $\mathrm{MB} \mathrm{NH} \mathrm{NH}_{2}$ resin $(140-170 \mu \mathrm{m}, 0.3 \mathrm{mmol} / \mathrm{g})$ was purchased from Rapp-Polymere (Tuebingen, German). The linkers (Fmoc-( $R$ )-3-amino-3-(2-nitrophenyl) propionic acid) and Fmoc6-aminohexanoic acid were purchased from Chem-Impex (Wood Dale, IL). All other materials were purchased from commercial sources and used without further purification unless otherwise noted.

Butylamine, histamine, and piperonylamine were incorporated without protecting groups. $N$-bocethylenediamine was purchased and used as received. Cyesteamine was protectected with a trityl group as reported by Maltese. ${ }^{2} \beta$-alanine and glycine were purchased as hydrochloride salts of the respective $t$-butyl esters. They were treated with $1 \mathrm{M} \mathrm{NaOH}$ solution saturated with $\mathrm{NaCl}$ and extracted with a mixture of 15:85 isopropanol:chloroform (v/v). The organic layers were dried over sodium sulfate and the solvent removed by evaporation to give the free bases.

Tentagel $\mathrm{MB} \mathrm{NH}$ resin was swollen in dichloromethane (DCM) before peptoid synthesis. Fmoc solid-phase synthesis was used to incorporate the first four linker members using HCTU as a coupling agent. The resin was split evenly into seven fritted columns, then acylation and addition of the first amine were performed as developed by Zuckermann et. al. ${ }^{3}$ For each acylation step, the resin beads were exposed to a solution of chloroacetic acid in dimethylformamide (DMF) (0.4 mM) as well as a solution of diisopropylcarbodiimide in $\operatorname{DMF}(2 \mathrm{M})$ with gentle agitation for 5 min on a nutator. The solutions were removed by filtration, and the resin rinsed with DMF. The resin beads 
were then exposed to a solution of amine in $\operatorname{DMF}(2 \mathrm{M})$ with gentle agitation for $2 \mathrm{~h}$ on a nutator. The solutions were removed by filtration, then all the resin was combined and mixed in DCM for 5 min. These steps were repeated three more times to add three more amines. After the resin was combined the last time, a solution of 4-methylpiperidine in DMF (20\%) was added to the resin to remove any acyl groups from imidazoles. The resin was then filtered and rinsed with DMF. The protecting groups were removed by incubation with a cleavage solution of 95:2.5:2.5 trifluoroacetic acid:water:triisopropylsilane for $1.5 \mathrm{~h}$, then rinsed with DCM, dried under vacuum, and refrigerated until use.

\section{Screening and sequencing}

Before incubating in the screening solution, a $40 \mathrm{mg}$ portion of the resin was swelled in water overnight. The screening buffer $(7 \mathrm{~mL})$ was added, consisting of $2 \mathrm{mM} \mathrm{UO}_{2}(\mathrm{OAc})_{2}, 1 \mathrm{M} \mathrm{NaCl}, 1 \mathrm{M}$ $\mathrm{MgSO}_{4}, 20 \mathrm{mM} \mathrm{NaHCO}_{3}$, adjusted to $\mathrm{pH}$ 7, and incubated for $1 \mathrm{~h}$. The library was then rinsed with water $(3 \times 1 \mathrm{~mL})$ to remove ions not bound to the ligands, then with ethanol $(1 \mathrm{~mL})$ to remove excess water. The resin was then transferred to a Petri dish. No color changes were observed after incubation. The dye solution was prepared by diluting $50 \mu \mathrm{L}$ of an aqueous $1 \mathrm{mM}$ solution of arsenazo III sodium salt into $1 \mathrm{~mL}$ ethanol. This was then added to the beads and allowed to sit for 1 min before removal of the excess dye solution. Upon evaporation of the remaining solvent, the dye would be trapped in the resin, binding to the metal if present. The resin in the Petri dish was then examined using a Leica S6D Microscope (Leica, Germany). The 15 individual beads with the most intense blue-green color were selected for ligand identification.

To avoid radiological contamination and ensure proper sequencing, the metal ions were removed by placing the selected beads on the membrane of a centrifugal filter unit. Amberlite cation exchange resin $\left(\mathrm{Na}^{+}\right.$form, $\left.0.5 \mathrm{mg}\right)$ was added to the filtrate collection tube of the filter unit, to which $1 \mathrm{M} \mathrm{HCl}$ $(0.5 \mathrm{~mL})$ was added. The tube was agitated gently on a nutator for $15 \mathrm{~min}$, followed by centrifugation. The acid solution was then removed, and water was added $(0.5 \mathrm{~mL})$. The tube was again gently agitated on a nutator for $15 \mathrm{~min}$, followed by centrifugation and removal of the water. The water rinse was repeated 3 times. The beads were then placed in a Petri dish with ethanol $(0.5$ $\mathrm{mL})$, then individually selected and placed into individual Eppendorf tubes. Ethanol $(5 \mu \mathrm{L})$ was added to each. The tubes were then placed in a computer controlled photoreactor with UVA bulbs for $8 \mathrm{~h}$ to cleave the peptoids from the resin beads.

After photocleavage, the ethanol was removed via evaporation and a solution $(0.5 \mu \mathrm{L})$ consisting of 1:1 water:acetonitrile with tris(2-carboxyethyl)phosphine $(0.5 \mathrm{mM})$. This solution was then mixed with matrix solution $(0.5 \mu \mathrm{L})$ consisting of $1: 1$ water:acetonitrile with $0.1 \%$ trifluoroacetic acid, 0.6 $\mathrm{M}$ ammonium phosphate, and $\alpha$-cyano-4-hydroxycinnamic acid $(5 \mathrm{mg})$ and spotted on a stainless steel MALDI plate. MALDI-TOF MS (Voyager- DE instrument, Applied Biosystems) was used to identify the selected sequences by mass, and then and MALDI-TOF-TOF MS/MS (AB Sciex 
TF4800, Applied Biosystems) was used to sequence the peptoids by fragmentation. MALDI spectra are shown in Figure S1.

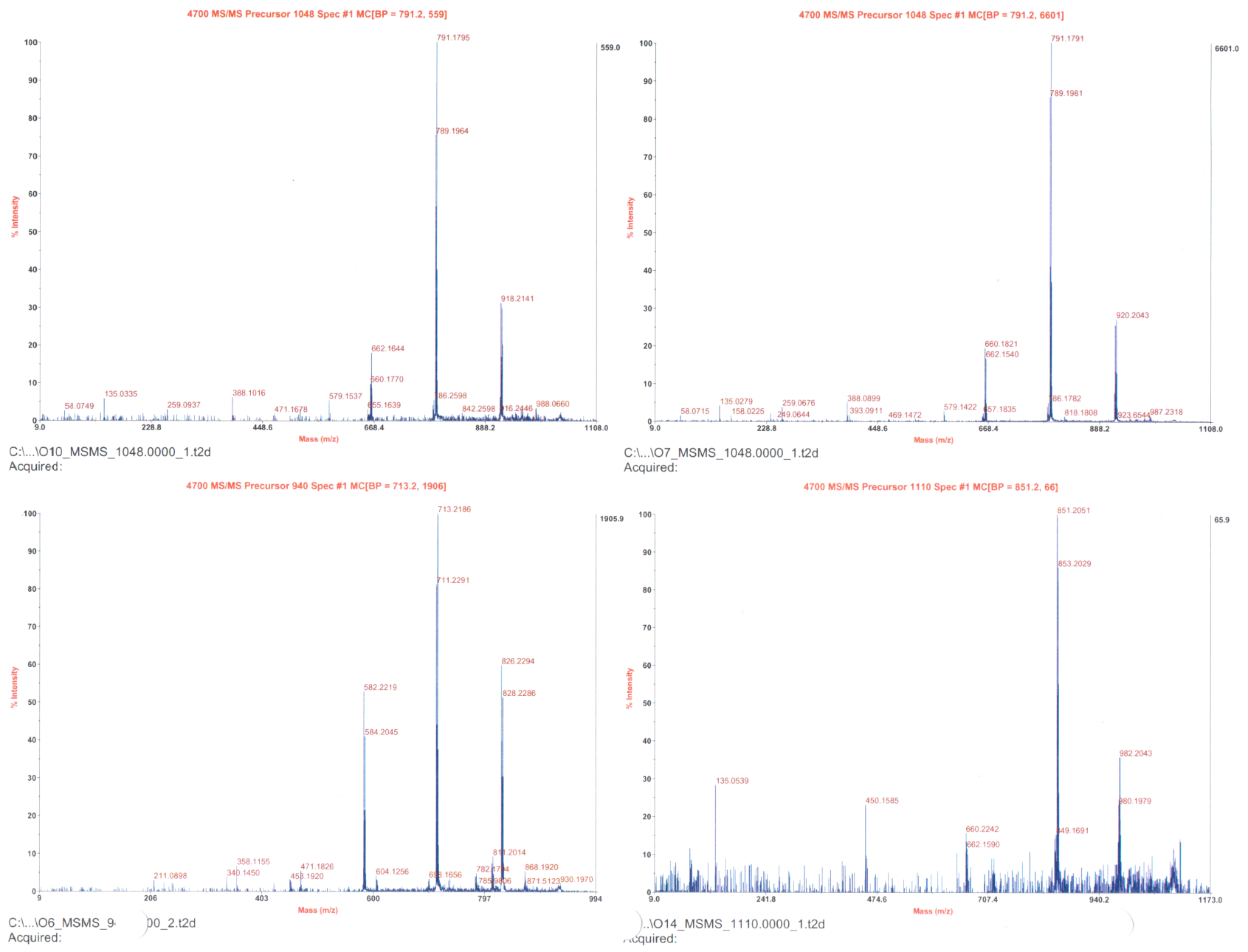

Figure S1: MALDI-TOF-TOF spectra for peptoids found to bind uranyl

\section{Fluorescence titration}

For preparative scale synthesis, the sequences were prepared analogously, using Fmoc-Rink Amide MBHA resin (Anaspec, Fremont, CA) instead of Tentagel $\mathrm{MB} \mathrm{NH}_{2}$ resin. The resin was treated with 20\% 4-methylpiperidine in DMF to cleave the initial fluorenylmethyl (Fmoc) group, followed by a cleavage cocktail of 95:2.5:2.5 trifluoroacetic acid:water:triisopropylsilane to remove the peptoids from the resin and cleave the protecting groups from the submonomers. The trifluoroaceitc acid was removed by evaporation and the peptoids precipitated from diethyl ether, then suspended in water. They were then purified by reverse-phase HPLC on a semi-preparatory HPLC column (Agilent). The fractions were then concentrated by speed vacuum and lyophilized. 
A series of 2-20 $\mu \mathrm{L}$ portions of $10 \mathrm{mM}$ or $80 \mathrm{mM}$ solutions of $\mathrm{UO}_{2}\left(\mathrm{NO}_{3}\right)_{2}$ were added to each peptoid sample $(100 \mu \mathrm{M}, 1.00 \mathrm{~mL})$ containing $20 \mathrm{mM}$ HEPES buffer at $\mathrm{pH}$ 7. The fluorescence was then measured at each increment using a Photon Technology International Quanta Master 4 Lformat scanning spectrofluorimeter with a LPS-220B 75W xenon lamp, using a $1 \mathrm{~cm}$ path length, $1.5 \mathrm{~mL}$ quartz cuvette. The excitation wavelength used was $200 \mathrm{~nm}$, and emission was measured over 300-550 nm wavelengths, with the maximum emission at $321 \mathrm{~nm}$, with $1 \mathrm{~nm}$ wavelength increments and $1 \mathrm{~s}$ integration times were used for all in. Concurrently, the uranyl fluorescence emission grew in at $369 \mathrm{~nm}$, and at high uranyl concentrations $(>1500 \mu \mathrm{M})$ it overlapped with the piperonyl emission. The emission was plotted against the concentration of uranyl and fit to a logistic binding curve (Equation 1). A1 and A2 are the asymptotes of the data, p is the slope of the curve, and $\mathrm{x} 0$ is the inflection point used to approximate the dissociation constant.

$$
y=\frac{\left(A_{1}-A_{2}\right)}{\left(1+\frac{x}{x_{0}}\right)^{p}}+A_{2}
$$

Equation S1

\section{Computations}

\section{Conformational analysis}

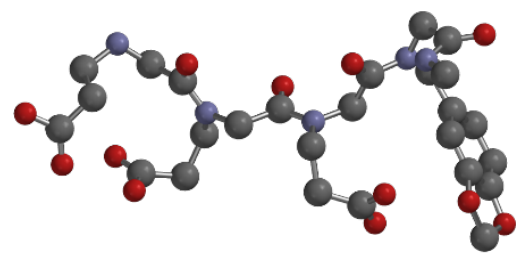

conf 1

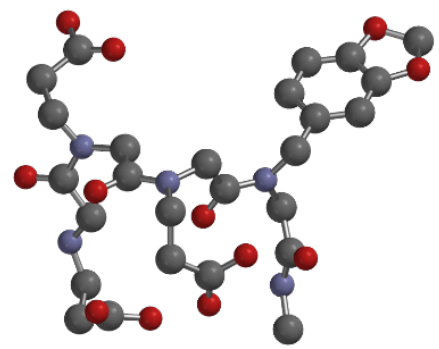

$\operatorname{conf} 2$

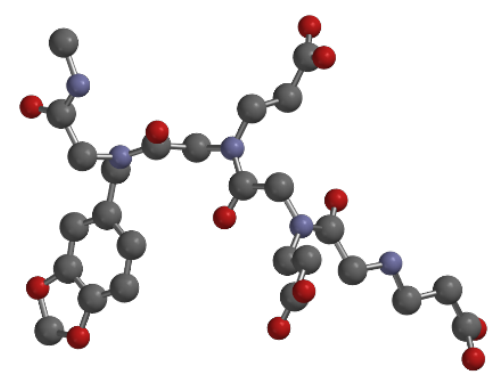

conf 3

Figure S2: Representative conformations for peptoid sequence $\boldsymbol{a}$. Conformation I was used for calculations of free energies of binding uranyl in solution in equation 2 . 


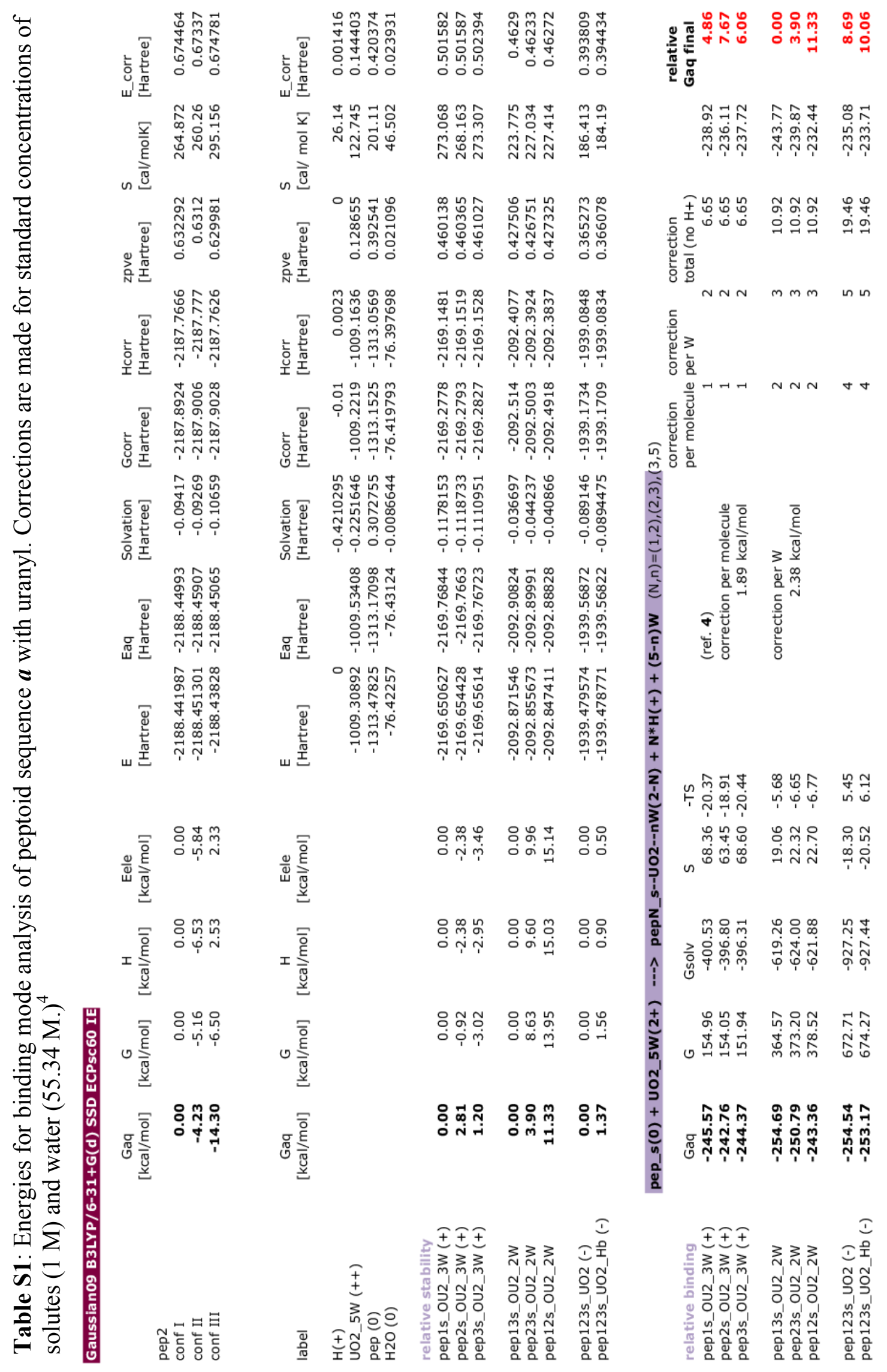


a1

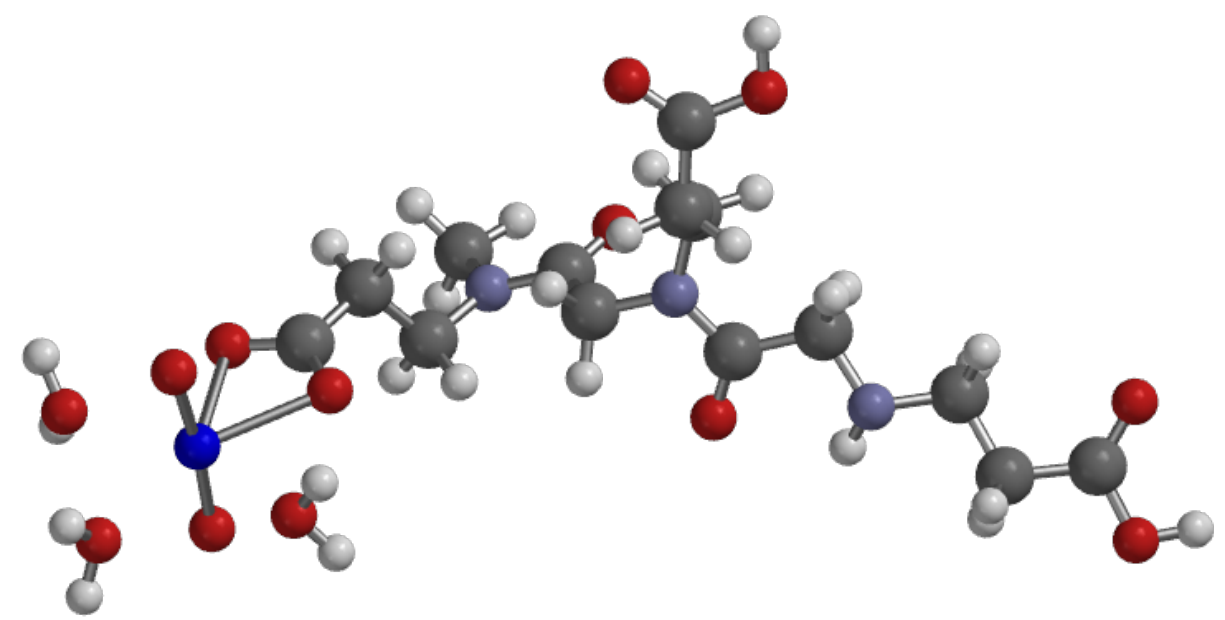

$\begin{array}{lrrr}\mathrm{C} & -1.18823 & -1.61654 & -1.76942 \\ \mathrm{O} & -1.03178 & -1.42389 & -2.96799 \\ \mathrm{~N} & -2.33392 & -2.19556 & -1.261 \\ \mathrm{C} & -0.09726 & -1.20103 & -0.75587 \\ \mathrm{H} & 0.215932 & -2.06289 & -0.16209 \\ \mathrm{H} & -0.49303 & -0.43726 & -0.07354 \\ \mathrm{C} & -2.57288 & -2.42157 & 0.151991 \\ \mathrm{H} & -3.07293 & -3.39181 & 0.263007 \\ \mathrm{C} & -3.39607 & -2.59531 & -2.18591 \\ \mathrm{H} & -3.03146 & -2.47026 & -3.20471 \\ \mathrm{H} & -4.29419 & -1.97842 & -2.05486 \\ \mathrm{H} & -3.6588 & -3.64661 & -2.0187 \\ \mathrm{C} & 2.107472 & -1.57286 & -1.65604 \\ \mathrm{O} & 2.052634 & -2.73662 & -1.25772 \\ \mathrm{~N} & 1.082716 & -0.68605 & -1.41975 \\ \mathrm{C} & 3.314754 & -1.08296 & -2.45508 \\ \mathrm{H} & 3.009167 & -1.07787 & -3.52265 \\ \mathrm{H} & 3.574818 & -0.04851 & -2.20131 \\ \mathrm{C} & 1.025336 & 0.680582 & -1.93652 \\ \mathrm{H} & -0.00527 & 0.875394 & -2.24708 \\ \mathrm{H} & 1.632863 & 0.766082 & -2.8382 \\ \mathrm{C} & 1.473172 & 1.720461 & -0.87958 \\ \mathrm{H} & 0.938941 & 1.5537 & 0.059686 \\ \mathrm{H} & 2.5476 & 1.619741 & -0.69351 \\ \mathrm{C} & 1.156415 & 3.129155 & -1.33149 \\ \mathrm{O} & 0.212453 & 3.789529 & -0.9495 \\ \mathrm{O} & 2.036968 & 3.569959 & -2.26116 \\ \mathrm{H} & -1.62842 & -2.48619 & 0.692569 \\ \mathrm{~N} & 4.455111 & -1.93957 & -2.19408\end{array}$




$\begin{array}{lrrr}\mathrm{H} & 4.126476 & -2.89717 & -2.07748 \\ \mathrm{C} & 5.509544 & -1.83621 & -3.19858 \\ \mathrm{H} & 5.848351 & -0.79497 & -3.24585 \\ \mathrm{H} & 5.155828 & -2.09468 & -4.21347 \\ \mathrm{C} & 6.687497 & -2.73194 & -2.81791 \\ \mathrm{H} & 7.033875 & -2.48694 & -1.80508 \\ \mathrm{H} & 6.395462 & -3.7898 & -2.78892 \\ \mathrm{C} & 7.859376 & -2.58701 & -3.7626 \\ \mathrm{O} & 8.812517 & -3.52216 & -3.5216 \\ \mathrm{O} & 7.976325 & -1.74573 & -4.62919 \\ \mathrm{C} & -3.45876 & -1.31466 & 0.800335 \\ \mathrm{H} & -4.44078 & -1.26987 & 0.323615 \\ \mathrm{H} & -2.96379 & -0.34424 & 0.677044 \\ \mathrm{C} & -3.64245 & -1.55761 & 2.268148 \\ \mathrm{O} & -4.78411 & -1.84405 & 2.775413 \\ \mathrm{O} & -2.64454 & -1.51017 & 3.07573 \\ \mathrm{O} & -3.66128 & -3.76469 & 4.837367 \\ \mathrm{U} & -3.94479 & -2.0278 & 4.990917 \\ \mathrm{O} & -4.25658 & -0.3283 & 5.356377 \\ \mathrm{O} & -1.57246 & -1.74055 & 5.78765 \\ \mathrm{H} & -1.15078 & -0.86375 & 5.749472 \\ \mathrm{O} & -4.23491 & -2.45054 & 7.470581 \\ \mathrm{H} & -4.16844 & -3.31025 & 7.921201 \\ \mathrm{H} & -4.4361 & -1.78244 & 8.148642 \\ \mathrm{O} & -6.42337 & -2.46294 & 5.100126 \\ \mathrm{H} & -7.04298 & -1.73818 & 4.902605 \\ \mathrm{H} & -6.79131 & -3.25902 & 4.677156 \\ \mathrm{H} & -0.88996 & -2.38431 & 5.527205 \\ \mathrm{H} & 1.762201 & 4.466733 & -2.53445\end{array}$


a2

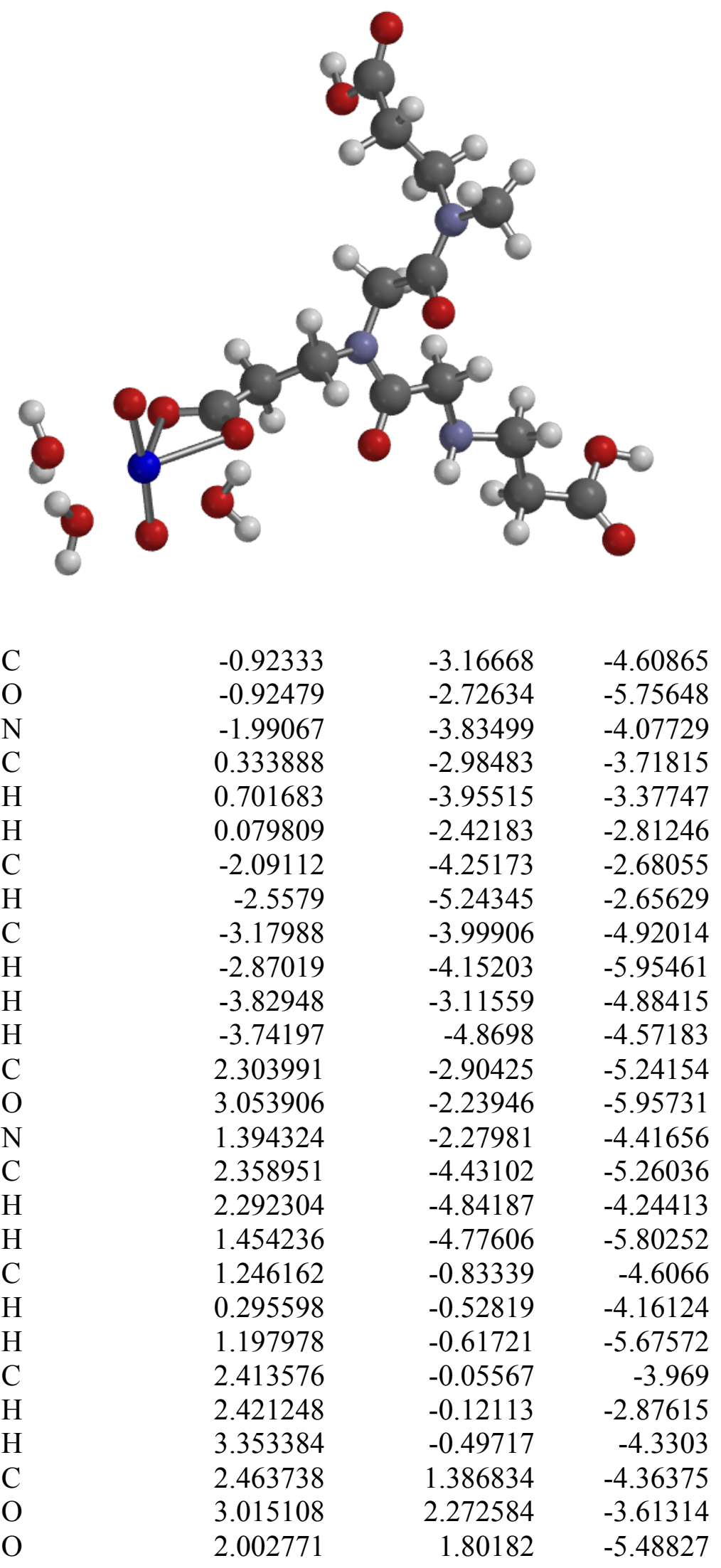




$\begin{array}{lrrr}\mathrm{H} & -1.09524 & -4.36228 & -2.25086 \\ \mathrm{~N} & 3.597785 & -4.87407 & -5.86869 \\ \mathrm{C} & 3.554787 & -6.24942 & -6.35473 \\ \mathrm{H} & 2.771304 & -6.4019 & -7.12199 \\ \mathrm{H} & 3.310886 & -6.90929 & -5.51442 \\ \mathrm{C} & 4.925191 & -6.64237 & -6.92709 \\ \mathrm{H} & 5.21607 & -5.96922 & -7.73903 \\ \mathrm{H} & 5.675317 & -6.55998 & -6.13087 \\ \mathrm{C} & 4.958043 & -8.04837 & -7.48398 \\ \mathrm{O} & 4.593918 & -8.97303 & -6.55537 \\ \mathrm{O} & 5.271593 & -8.35495 & -8.61424 \\ \mathrm{C} & -2.92304 & -3.2702 & -1.82008 \\ \mathrm{H} & -3.94491 & -3.19407 & -2.19839 \\ \mathrm{H} & -2.46209 & -2.27555 & -1.85695 \\ \mathrm{C} & -3.00092 & -3.71958 & -0.37578 \\ \mathrm{O} & -3.99882 & -4.09465 & 0.196302 \\ \mathrm{O} & -1.78057 & -3.67176 & 0.225187 \\ \mathrm{O} & 1.208118 & 4.553465 & -4.51251 \\ \mathrm{U} & 2.750193 & 4.01644 & -5.1896 \\ \mathrm{O} & 4.321026 & 3.701621 & -5.93365 \\ \mathrm{O} & 3.94231 & 5.001897 & -3.19869 \\ \mathrm{H} & 4.839518 & 4.685245 & -2.99126 \\ \mathrm{O} & 3.033907 & 6.399348 & -6.00312 \\ \mathrm{H} & 2.404355 & 7.131116 & -5.88294 \\ \mathrm{H} & 3.778439 & 6.739733 & -6.52874 \\ \mathrm{O} & 1.704719 & 3.842023 & -7.47935 \\ \mathrm{H} & 2.135248 & 3.242472 & -8.11529 \\ \mathrm{H} & 0.750812 & 3.656845 & -7.54258 \\ \mathrm{H} & 3.473203 & 5.06642 & -2.34805 \\ \mathrm{H} & -1.89297 & -3.98404 & 1.143995 \\ \mathrm{H} & 4.652199 & -9.84898 & -6.98294\end{array}$


a3

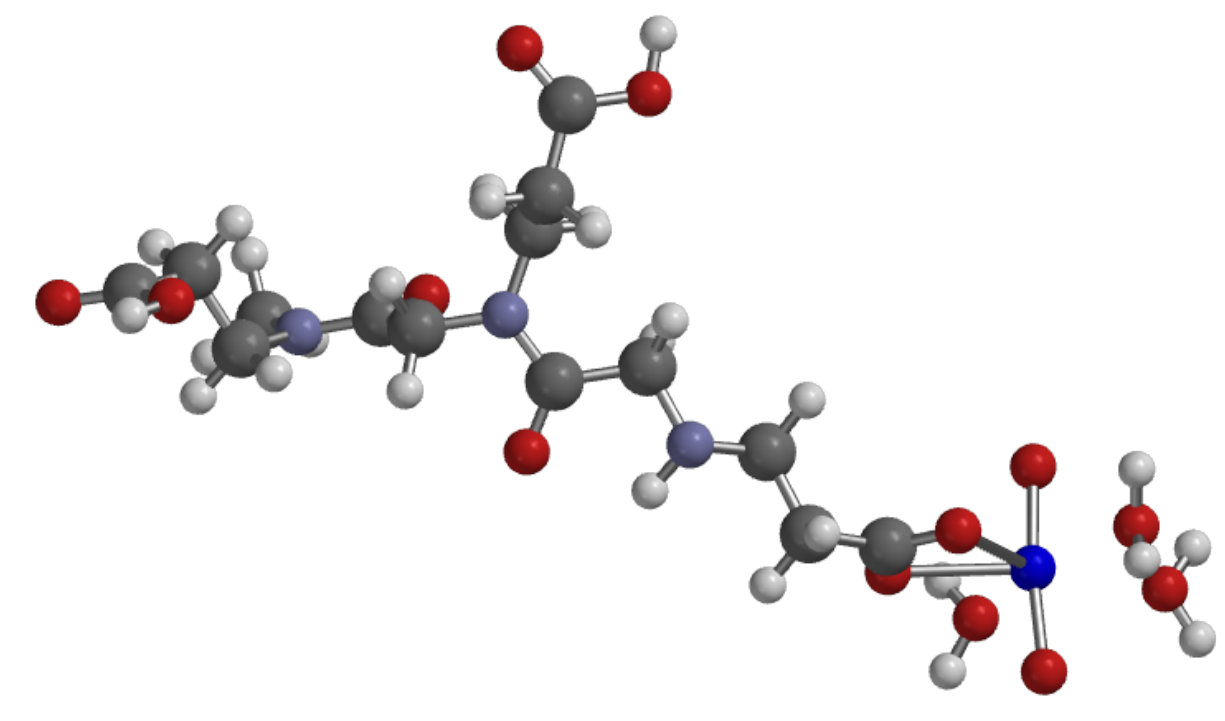

$\begin{array}{lrrr}\mathrm{C} & 4.951096 & 4.896685 & -7.82328 \\ \mathrm{O} & 5.515047 & 3.861822 & -8.17501 \\ \mathrm{~N} & 4.171252 & 4.958586 & -6.70181 \\ \mathrm{C} & 5.115542 & 6.192622 & -8.65374 \\ \mathrm{H} & 5.53065 & 6.990699 & -8.03477 \\ \mathrm{H} & 4.14531 & 6.528739 & -9.03692 \\ \mathrm{C} & 3.384531 & 6.123441 & -6.30892 \\ \mathrm{H} & 3.459545 & 6.232528 & -5.22053 \\ \mathrm{C} & 3.993713 & 3.728888 & -5.92493 \\ \mathrm{H} & 4.919589 & 3.152772 & -5.94669 \\ \mathrm{H} & 3.190463 & 3.102057 & -6.33246 \\ \mathrm{H} & 3.749658 & 3.995141 & -4.89268 \\ \mathrm{C} & 7.348007 & 6.201232 & -9.56023 \\ \mathrm{O} & 7.774991 & 6.670721 & -8.5037 \\ \mathrm{~N} & 6.01705 & 5.978885 & -9.77387 \\ \mathrm{C} & 8.334623 & 5.828509 & -10.6757 \\ \mathrm{H} & 8.439479 & 4.726193 & -10.6505 \\ \mathrm{H} & 7.946105 & 6.092016 & -11.6662 \\ \mathrm{C} & 5.473771 & 5.307393 & -10.9542 \\ \mathrm{H} & 4.697191 & 4.614935 & -10.6168 \\ \mathrm{H} & 6.24326 & 4.692537 & -11.4234 \\ \mathrm{C} & 4.887663 & 6.310197 & -11.9775 \\ \mathrm{H} & 4.126459 & 6.932712 & -11.5002 \\ \mathrm{H} & 5.684336 & 6.95835 & -12.3571 \\ \mathrm{C} & 4.227408 & 5.579298 & -13.1257 \\ \mathrm{O} & 3.04049 & 5.361413 & -13.2327 \\ \mathrm{O} & 5.138402 & 5.139477 & -14.0348 \\ \mathrm{H} & 3.813266 & 7.025311 & -6.74696 \\ \mathrm{~N} & 9.595675 & 6.51671 & -10.4374 \\ \mathrm{H} & 9.656202 & 6.707208 & -9.43585 \\ & & & \\ & & & \end{array}$




$\begin{array}{lrrr}\mathrm{C} & 10.76444 & 5.81189 & -10.9275 \\ \mathrm{H} & 10.63715 & 5.619975 & -12.0007 \\ \mathrm{H} & 10.9251 & 4.832764 & -10.437 \\ \mathrm{C} & 12.01277 & 6.704976 & -10.7181 \\ \mathrm{H} & 11.88752 & 7.638041 & -11.2729 \\ \mathrm{H} & 12.11536 & 6.932027 & -9.65038 \\ \mathrm{C} & 13.26211 & 6.018927 & -11.1693 \\ \mathrm{O} & 13.80186 & 6.263947 & -12.3093 \\ \mathrm{O} & 13.84236 & 5.128964 & -10.4447 \\ \mathrm{C} & 1.892293 & 6.00592 & -6.70995 \\ \mathrm{H} & 1.437324 & 5.134801 & -6.23259 \\ \mathrm{H} & 1.812944 & 5.895849 & -7.79715 \\ \mathrm{C} & 1.118132 & 7.229279 & -6.2693 \\ \mathrm{O} & 0.38974 & 7.295245 & -5.30386 \\ \mathrm{O} & 1.365605 & 8.294329 & -7.07781 \\ \mathrm{O} & 14.68375 & 3.408054 & -12.7948 \\ \mathrm{U} & 15.56421 & 4.720261 & -12.0034 \\ \mathrm{O} & 16.63737 & 5.926298 & -11.2847 \\ \mathrm{O} & 15.90915 & 5.814027 & -14.2501 \\ \mathrm{H} & 16.12264 & 6.763757 & -14.2754 \\ \mathrm{O} & 17.676 & 3.540184 & -12.759 \\ \mathrm{H} & 17.7234 & 2.709423 & -13.2629 \\ \mathrm{H} & 18.58908 & 3.83158 & -12.5924 \\ \mathrm{O} & 15.97546 & 3.217486 & -10.0192 \\ \mathrm{H} & 16.19372 & 3.629114 & -9.16412 \\ \mathrm{H} & 15.32854 & 2.51636 & -9.82391 \\ \mathrm{H} & 15.24603 & 5.667287 & -14.9479 \\ \mathrm{H} & 0.855749 & 9.051523 & -6.73027\end{array}$


$a 12$

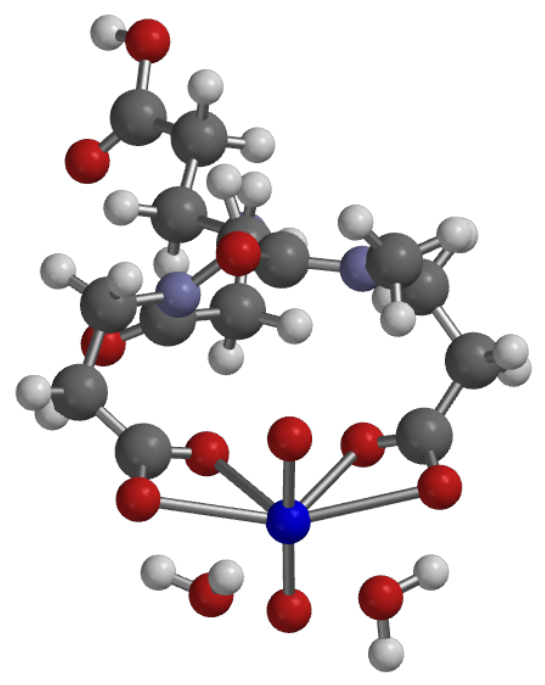

$\begin{array}{lrrr}\mathrm{C} & -0.75142 & 3.480891 & 0.733919 \\ \mathrm{O} & -1.8516 & 3.929161 & 1.043097 \\ \mathrm{~N} & 0.280138 & 4.2941 & 0.325887 \\ \mathrm{C} & -0.46431 & 1.962624 & 0.80974 \\ \mathrm{H} & 0.384547 & 1.788043 & 1.478552 \\ \mathrm{H} & -0.14063 & 1.589584 & -0.1637 \\ \mathrm{C} & 1.56232 & 3.800128 & -0.17101 \\ \mathrm{H} & 2.344084 & 4.49546 & 0.152231 \\ \mathrm{C} & 0.018688 & 5.732918 & 0.23666 \\ \mathrm{H} & 0.950638 & 6.276451 & 0.41785 \\ \mathrm{H} & -0.72176 & 6.005361 & 0.989616 \\ \mathrm{H} & -0.37628 & 6.015144 & -0.74747 \\ \mathrm{C} & -2.64133 & 0.826945 & 0.457065 \\ \mathrm{O} & -3.72287 & 0.447973 & 0.892111 \\ \mathrm{~N} & -1.59335 & 1.191501 & 1.287953 \\ \mathrm{C} & -2.38192 & 0.880425 & -1.06745 \\ \mathrm{H} & -2.23305 & 1.928539 & -1.35962 \\ \mathrm{H} & -1.44499 & 0.360677 & -1.29533 \\ \mathrm{C} & -1.77659 & 1.059609 & 2.737137 \\ \mathrm{H} & -2.66861 & 0.448212 & 2.869478 \\ \mathrm{H} & -1.97117 & 2.041637 & 3.188497 \\ \mathrm{C} & -0.57277 & 0.40248 & 3.457164 \\ \mathrm{H} & 0.267645 & 1.089435 & 3.59946 \\ \mathrm{H} & -0.90856 & 0.108078 & 4.460752 \\ \mathrm{C} & -0.06464 & -0.83193 & 2.738807 \\ \mathrm{O} & -0.89717 & -1.62946 & 2.207552 \\ \mathrm{O} & 1.183795 & -1.03927 & 2.567583 \\ \mathrm{H} & 1.785534 & 2.838735 & 0.29425 \\ \mathrm{~N} & -3.44121 & 0.347134 & -1.91093\end{array}$




$\begin{array}{lrrr}\mathrm{H} & -4.29817 & 0.85788 & -1.71141 \\ \mathrm{C} & -3.75062 & -1.09183 & -1.83808 \\ \mathrm{H} & -3.96946 & -1.42919 & -0.81728 \\ \mathrm{H} & -4.66051 & -1.22301 & -2.43402 \\ \mathrm{C} & -2.6608 & -1.9933 & -2.44874 \\ \mathrm{H} & -2.20301 & -1.53007 & -3.33083 \\ \mathrm{H} & -3.12071 & -2.92985 & -2.79618 \\ \mathrm{C} & -1.5687 & -2.39776 & -1.48422 \\ \mathrm{O} & -0.44219 & -2.85639 & -1.87994 \\ \mathrm{O} & -1.74751 & -2.26083 & -0.23267 \\ \mathrm{C} & 1.643055 & 3.614631 & -1.70556 \\ \mathrm{H} & 2.577237 & 3.080652 & -1.92678 \\ \mathrm{H} & 0.813224 & 3.00496 & -2.07159 \\ \mathrm{O} & 0.432656 & -4.07082 & 1.043821 \\ \mathrm{U} & 0.532372 & -2.405 & 0.434225 \\ \mathrm{O} & 0.797626 & -0.78858 & -0.25353 \\ \mathrm{C} & 1.660535 & 4.89478 & -2.51283 \\ \mathrm{O} & 0.909799 & 5.170459 & -3.42346 \\ \mathrm{O} & 2.660799 & 5.729706 & -2.11786 \\ \mathrm{H} & 2.623963 & 6.516222 & -2.69497 \\ \mathrm{H} & 2.834144 & -1.92 & 2.073413 \\ \mathrm{O} & 2.981959 & -2.54619 & 1.334638 \\ \mathrm{H} & 3.204945 & -3.39653 & 1.746457 \\ \mathrm{H} & 2.454297 & -4.22251 & -1.33682 \\ \mathrm{O} & 2.103383 & -3.32215 & -1.42244 \\ \mathrm{H} & 1.437165 & -3.34891 & -2.13905\end{array}$




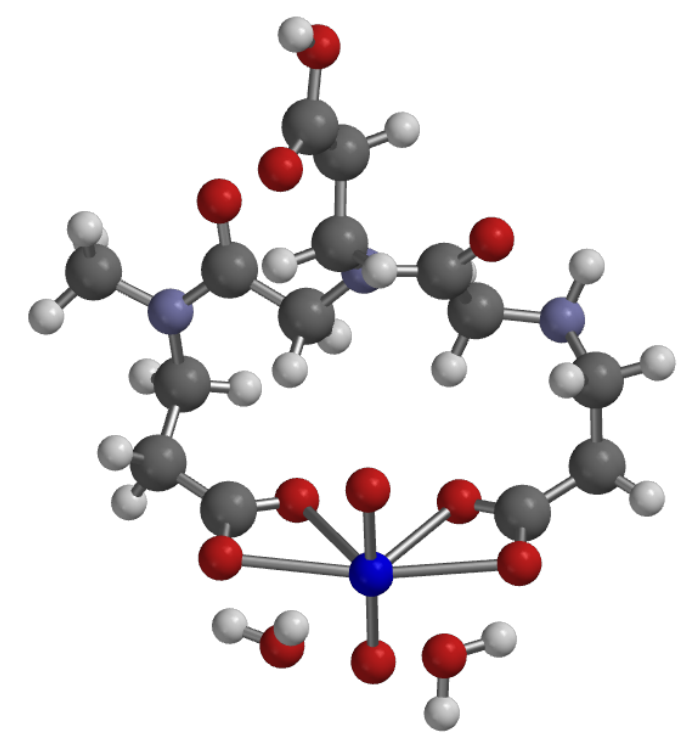

$\begin{array}{lrrr}\mathrm{C} & -1.51273 & -3.30237 & 0.987562 \\ \mathrm{O} & -1.38655 & -4.47625 & 0.646917 \\ \mathrm{~N} & -2.68645 & -2.83427 & 1.536302 \\ \mathrm{C} & -0.40946 & -2.25937 & 0.726664 \\ \mathrm{H} & -0.1137 & -1.79788 & 1.667703 \\ \mathrm{H} & -0.83952 & -1.45592 & 0.114775 \\ \mathrm{C} & -2.83797 & -1.5863 & 2.293687 \\ \mathrm{H} & -3.46493 & -1.82462 & 3.15963 \\ \mathrm{C} & -3.80447 & -3.77312 & 1.639763 \\ \mathrm{H} & -3.81314 & -4.27905 & 2.615491 \\ \mathrm{H} & -3.71734 & -4.52884 & 0.859615 \\ \mathrm{H} & -4.74541 & -3.22619 & 1.517548 \\ \mathrm{C} & 2.049751 & -2.47417 & 0.417835 \\ \mathrm{O} & 3.007634 & -2.82731 & -0.27521 \\ \mathrm{~N} & 0.764876 & -2.79953 & 0.057021 \\ \mathrm{C} & 2.308727 & -1.67499 & 1.718052 \\ \mathrm{H} & 1.636613 & -0.82068 & 1.809336 \\ \mathrm{H} & 2.101695 & -2.33381 & 2.573672 \\ \mathrm{C} & 0.582617 & -3.36925 & -1.2923 \\ \mathrm{H} & 1.187146 & -2.78871 & -1.99613 \\ \mathrm{H} & -0.46565 & -3.24579 & -1.5681 \\ \mathrm{C} & 0.967401 & -4.84869 & -1.37702 \\ \mathrm{H} & 2.032854 & -4.98193 & -1.16461 \\ \mathrm{H} & 0.407751 & -5.42174 & -0.62977 \\ \mathrm{H} & -1.87468 & -1.26398 & 2.692566 \\ \mathrm{~N} & 3.678489 & -1.19089 & 1.832667 \\ \mathrm{H} & 4.303319 & -1.97389 & 1.654541 \\ \mathrm{C} & 4.067564 & -0.10163 & 0.926765\end{array}$




$\begin{array}{lrrr}\mathrm{H} & 5.158556 & -0.14503 & 0.848964 \\ \mathrm{H} & 3.678406 & -0.23213 & -0.09537 \\ \mathrm{C} & 3.719323 & 1.324154 & 1.449726 \\ \mathrm{H} & 4.443131 & 2.035895 & 1.040224 \\ \mathrm{H} & 3.795403 & 1.321647 & 2.542001 \\ \mathrm{C} & 2.345765 & 1.796344 & 1.047148 \\ \mathrm{O} & 1.303826 & 1.37991 & 1.648711 \\ \mathrm{O} & 2.179557 & 2.58148 & 0.047807 \\ \mathrm{C} & -3.47351 & -0.40817 & 1.513794 \\ \mathrm{H} & -4.07013 & -0.75702 & 0.665056 \\ \mathrm{H} & -4.15898 & 0.142402 & 2.173294 \\ \mathrm{C} & -2.46046 & 0.610657 & 1.024846 \\ \mathrm{O} & -1.35416 & 0.735962 & 1.636829 \\ \mathrm{O} & -2.69317 & 1.348346 & 0.012124 \\ \mathrm{O} & -0.666 & 3.649536 & 0.92899 \\ \mathrm{U} & -0.28428 & 2.183953 & 0.008456 \\ \mathrm{O} & 0.069611 & 0.774565 & -1.01128 \\ \mathrm{C} & 0.666793 & -5.42646 & -2.7358 \\ \mathrm{O} & 0.089662 & -4.86548 & -3.64764 \\ \mathrm{O} & 1.113874 & -6.70536 & -2.85247 \\ \mathrm{H} & 0.870006 & -7.00932 & -3.74737 \\ \mathrm{H} & 0.474845 & 4.688854 & -1.72035 \\ \mathrm{O} & 0.743128 & 3.759551 & -1.80881 \\ \mathrm{H} & 1.68025 & 3.709228 & -1.53539 \\ \mathrm{H} & -1.57764 & 2.696181 & -2.79165 \\ \mathrm{O} & -1.86854 & 2.971161 & -1.90665 \\ \mathrm{H} & -2.6687 & 2.453516 & -1.69384\end{array}$


a23

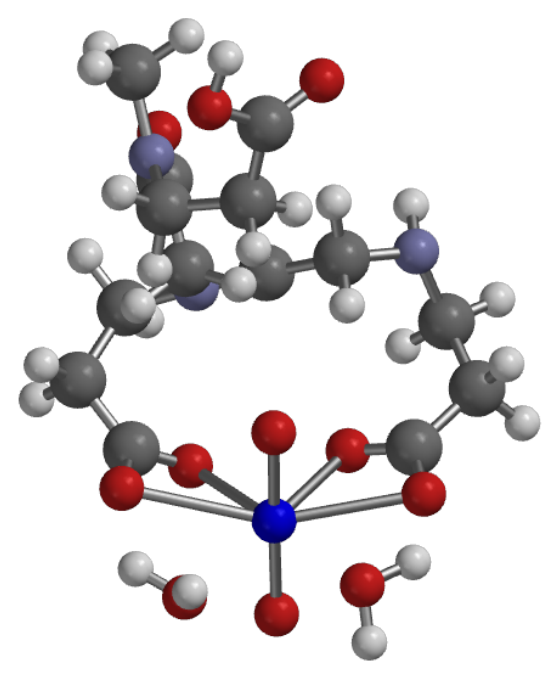

$\begin{array}{lrrr}\mathrm{C} & 2.3208 & -0.47976 & -0.86774 \\ \mathrm{O} & 2.449178 & 0.008713 & -1.99195 \\ \mathrm{~N} & 2.993045 & 0.052055 & 0.208852 \\ \mathrm{C} & 1.574946 & -1.82522 & -0.72739 \\ \mathrm{H} & 1.615362 & -2.19938 & 0.289648 \\ \mathrm{H} & 2.185489 & -2.50875 & -1.34092 \\ \mathrm{C} & 2.82595 & -0.28586 & 1.626559 \\ \mathrm{H} & 2.004537 & -0.99082 & 1.736259 \\ \mathrm{C} & 3.967678 & 1.108067 & -0.08678 \\ \mathrm{H} & 3.516401 & 2.104361 & -0.02635 \\ \mathrm{H} & 4.345576 & 0.969386 & -1.1 \\ \mathrm{H} & 4.794034 & 1.036402 & 0.628914 \\ \mathrm{C} & -0.76549 & -2.6463 & -0.53502 \\ \mathrm{O} & -1.82949 & -2.9325 & -1.09053 \\ \mathrm{~N} & 0.180371 & -1.88269 & -1.1821 \\ \mathrm{C} & -0.50556 & -3.15645 & 0.893032 \\ \mathrm{H} & 0.026924 & -2.41761 & 1.500414 \\ \mathrm{H} & -1.49713 & -3.27708 & 1.337356 \\ \mathrm{C} & -0.16965 & -1.4054 & -2.53397 \\ \mathrm{H} & -0.38983 & -2.27211 & -3.1701 \\ \mathrm{H} & 0.706463 & -0.8912 & -2.92588 \\ \mathrm{C} & -1.40799 & -0.46829 & -2.61663 \\ \mathrm{H} & -1.47879 & -0.07942 & -3.63715 \\ \mathrm{H} & -2.30509 & -1.06075 & -2.4069 \\ \mathrm{C} & -1.40465 & 0.684704 & -1.6484 \\ \mathrm{O} & -1.177 & 0.45331 & -0.41512 \\ \mathrm{O} & -1.59615 & 1.898197 & -1.99957 \\ \mathrm{H} & 3.744053 & -0.76884 & 1.99275 \\ \mathrm{~N} & 0.187582 & -4.44695 & 0.990428\end{array}$




$\begin{array}{lrrr}\mathrm{H} & 1.107497 & -4.3958 & 0.554813 \\ \mathrm{C} & -0.55266 & -5.59568 & 0.454211 \\ \mathrm{H} & -1.53018 & -5.62633 & 0.945431 \\ \mathrm{H} & -0.75192 & -5.52696 & -0.62662 \\ \mathrm{C} & 0.22014 & -6.87899 & 0.763721 \\ \mathrm{H} & 0.43359 & -6.93286 & 1.840247 \\ \mathrm{H} & 1.196298 & -6.89448 & 0.261532 \\ \mathrm{C} & 2.532192 & 0.932238 & 2.534806 \\ \mathrm{H} & 2.415441 & 0.556607 & 3.560523 \\ \mathrm{H} & 3.356996 & 1.649378 & 2.548273 \\ \mathrm{C} & 1.254216 & 1.645708 & 2.136618 \\ \mathrm{O} & 1.164623 & 2.915524 & 2.123607 \\ \mathrm{O} & 0.283467 & 0.944182 & 1.717156 \\ \mathrm{O} & -2.08888 & 2.883058 & 1.14419 \\ \mathrm{U} & -0.59358 & 2.650435 & 0.218778 \\ \mathrm{O} & 0.909726 & 2.602211 & -0.71709 \\ \mathrm{C} & -0.53044 & -8.1337 & 0.380493 \\ \mathrm{O} & -1.71259 & -8.21305 & 0.12169 \\ \mathrm{O} & 0.285354 & -9.22269 & 0.377813 \\ \mathrm{H} & -0.27034 & -9.99411 & 0.155442 \\ \mathrm{H} & -0.6975 & 5.531255 & 1.427388 \\ \mathrm{O} & 0.030939 & 5.064468 & 0.986073 \\ \mathrm{H} & 0.670918 & 4.817392 & 1.683094 \\ \mathrm{H} & -0.61017 & 5.027505 & -1.78852 \\ \mathrm{O} & -1.34423 & 4.489431 & -1.44978 \\ \mathrm{H} & -1.62293 & 3.901726 & -2.18042\end{array}$




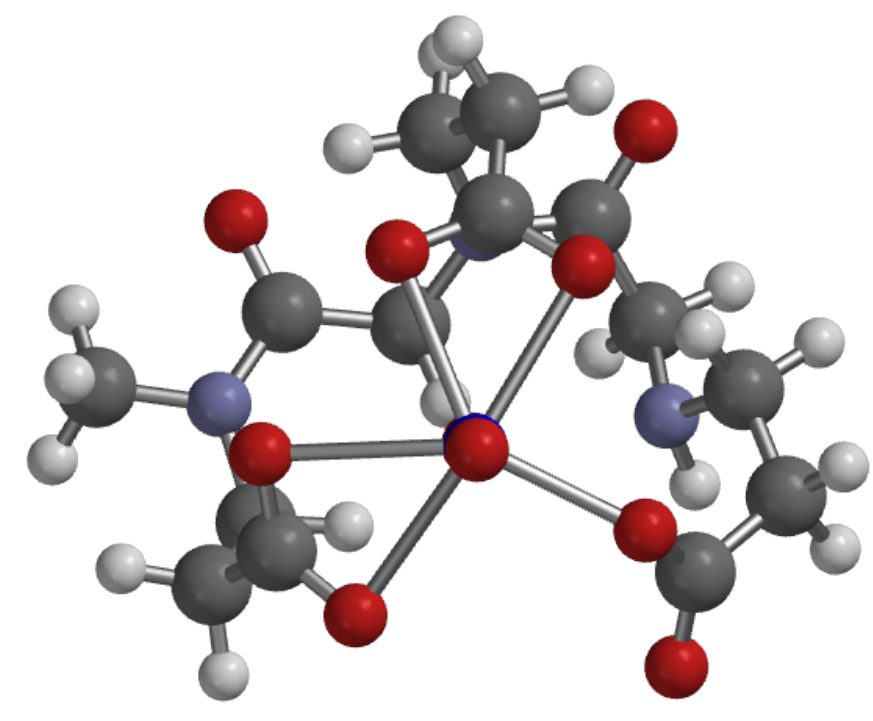

$\begin{array}{lrrr}\mathrm{C} & -1.68159 & -1.61463 & -1.92928 \\ \mathrm{O} & -1.7446 & -1.43689 & -3.15198 \\ \mathrm{~N} & -2.80852 & -1.51371 & -1.14517 \\ \mathrm{C} & -0.34869 & -2.06246 & -1.31317 \\ \mathrm{H} & -0.26312 & -3.10866 & -1.64223 \\ \mathrm{H} & -0.34389 & -2.0452 & -0.22873 \\ \mathrm{C} & -2.96675 & -1.8889 & 0.26677 \\ \mathrm{H} & -3.51092 & -2.84686 & 0.29656 \\ \mathrm{C} & -4.05682 & -1.1911 & -1.83737 \\ \mathrm{H} & -3.9233 & -1.35537 & -2.906 \\ \mathrm{H} & -4.33045 & -0.14282 & -1.67044 \\ \mathrm{H} & -4.86157 & -1.83571 & -1.46138 \\ \mathrm{C} & 2.09686 & -1.85935 & -1.54598 \\ \mathrm{O} & 3.10402 & -1.44427 & -2.13211 \\ \mathrm{~N} & 0.85526 & -1.33173 & -1.79014 \\ \mathrm{C} & 2.26563 & -2.96218 & -0.48033 \\ \mathrm{H} & 1.59751 & -3.80841 & -0.68511 \\ \mathrm{H} & 3.30397 & -3.31078 & -0.61701 \\ \mathrm{C} & 0.81288 & -0.1359 & -2.66165 \\ \mathrm{H} & -0.22688 & 0.1203 & -2.83231 \\ \mathrm{H} & 1.25907 & -0.39526 & -3.62868 \\ \mathrm{C} & 1.55913 & 1.10296 & -2.10942 \\ \mathrm{H} & 2.62522 & 0.90335 & -1.99892 \\ \mathrm{H} & 1.424 & 1.89855 & -2.85489 \\ \mathrm{C} & 0.98725 & 1.58471 & -0.79159 \\ \mathrm{O} & 1.69945 & 1.56606 & 0.26768 \\ \mathrm{O} & -0.23899 & 1.91174 & -0.71712 \\ \mathrm{H} & -1.99153 & -2.04773 & 0.72121 \\ \mathrm{~N} & 1.98821 & -2.48645 & 0.87475 \\ \mathrm{H} & 1.97902 & -3.27513 & 1.51859\end{array}$




$\begin{array}{lrrr}\mathrm{C} & 2.91728 & -1.45174 & 1.35095 \\ \mathrm{H} & 2.68462 & -0.5119 & 0.84322 \\ \mathrm{H} & 3.96392 & -1.70662 & 1.09196 \\ \mathrm{C} & 2.85776 & -1.2463 & 2.8741 \\ \mathrm{H} & 3.15551 & -2.16993 & 3.38659 \\ \mathrm{H} & 3.59532 & -0.47168 & 3.12076 \\ \mathrm{C} & 1.50278 & -0.8202 & 3.46019 \\ \mathrm{O} & 0.86317 & -1.59497 & 4.16627 \\ \mathrm{O} & 1.13158 & 0.41779 & 3.19423 \\ \mathrm{C} & -3.77128 & -0.88553 & 1.14545 \\ \mathrm{H} & -4.06458 & -1.42027 & 2.05442 \\ \mathrm{H} & -4.67961 & -0.56468 & 0.62594 \\ \mathrm{C} & -2.9417 & 0.32654 & 1.54127 \\ \mathrm{O} & -2.40043 & 0.36179 & 2.69642 \\ \mathrm{O} & -2.66517 & 1.20395 & 0.67156 \\ \mathrm{O} & -0.28862 & -0.39016 & 1.02701 \\ \mathrm{U} & -0.31503 & 1.25946 & 1.67928 \\ \mathrm{O} & -0.3623 & 2.90789 & 2.35961\end{array}$




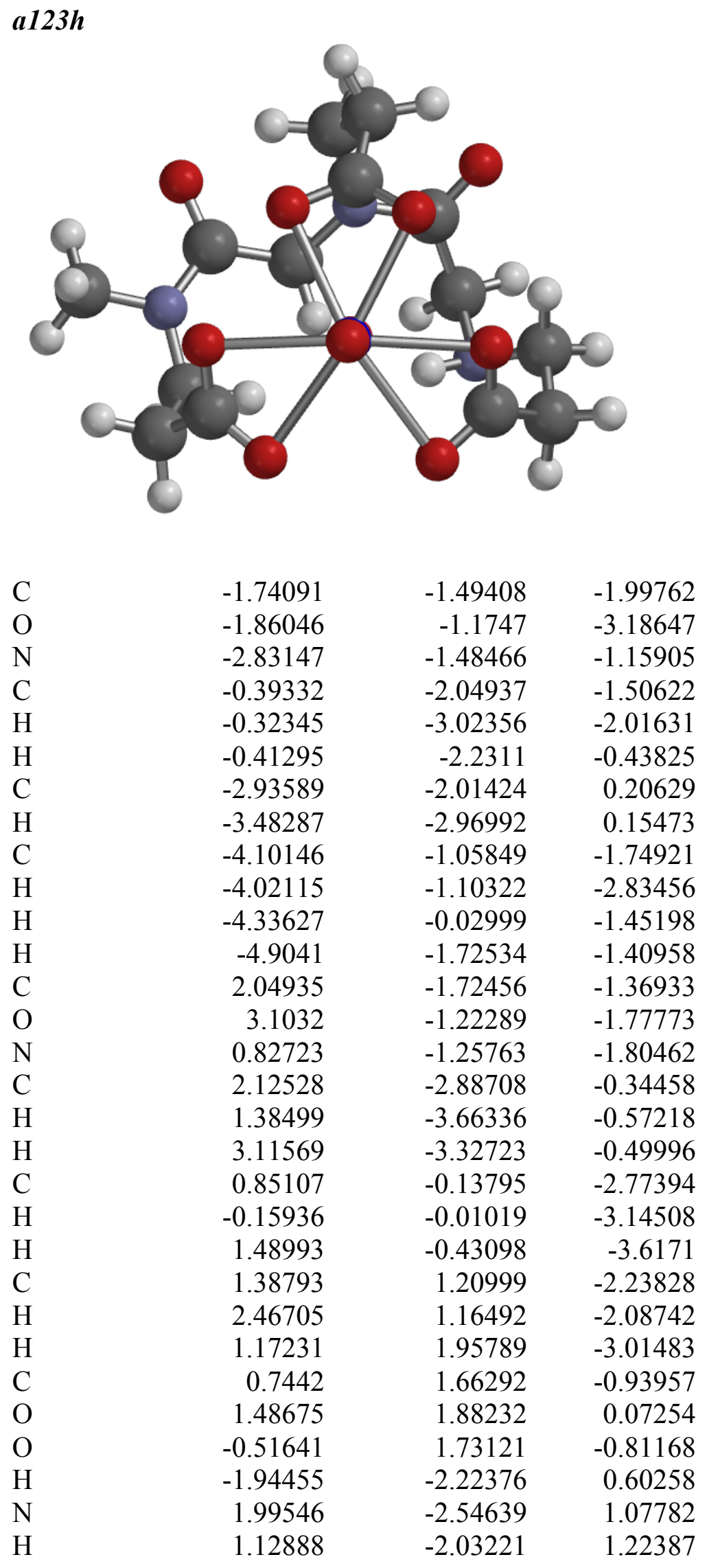




$\begin{array}{lrrr}\mathrm{C} & 3.10648 & -1.76636 & 1.64871 \\ \mathrm{H} & 3.35539 & -0.87101 & 1.06106 \\ \mathrm{H} & 3.99511 & -2.41339 & 1.65828 \\ \mathrm{C} & 2.79042 & -1.32486 & 3.09697 \\ \mathrm{H} & 2.43011 & -2.17168 & 3.68999 \\ \mathrm{H} & 3.71832 & -0.94576 & 3.5442 \\ \mathrm{C} & 1.76051 & -0.19775 & 3.12281 \\ \mathrm{O} & 0.61319 & -0.34485 & 3.63325 \\ \mathrm{O} & 2.03995 & 0.87265 & 2.48305 \\ \mathrm{C} & -3.68683 & -1.10874 & 1.22224 \\ \mathrm{H} & -3.91556 & -1.72594 & 2.09711 \\ \mathrm{H} & -4.62906 & -0.75044 & 0.79607 \\ \mathrm{C} & -2.82773 & 0.06605 & 1.66095 \\ \mathrm{O} & -2.19535 & 0.00471 & 2.76052 \\ \mathrm{O} & -2.62611 & 1.01893 & 0.84814 \\ \mathrm{O} & -0.07667 & -0.45858 & 0.91447 \\ \mathrm{U} & -0.24372 & 1.16027 & 1.65555 \\ \mathrm{O} & -0.46697 & 2.74976 & 2.42236\end{array}$




\section{Full citation for reference 34:}

Gaussian 09, Revision A.2, Frisch, M. J.; Trucks, G. W.; Schlegel, H. B.; Scuseria, G. E.; Robb, M. A.; Cheeseman, J. R.; Scalmani, G.; Barone, V.; Mennucci, B.; Petersson, G. A.; Nakatsuji, H.; Caricato, M.; Li, X.; Hratchian, H. P.; Izmaylov, A. F.; Bloino, J.; Zheng, G.; Sonnenberg, J. L.; Hada, M.; Ehara, M.; Toyota, K.; Fukuda, R.; Hasegawa, J.; Ishida, M.; Nakajima, T.; Honda, Y.; Kitao, O.; Nakai, H.; Vreven, T.; Montgomery, Jr., J. A.; Peralta, J. E.; Ogliaro, F.; Bearpark, M.; Heyd, J. J.; Brothers, E.; Kudin, K. N.; Staroverov, V. N.; Kobayashi, R.; Normand, J.; Raghavachari, K.; Rendell, A.; Burant, J. C.; Iyengar, S. S.; Tomasi, J.; Cossi, M.; Rega, N.; Millam, N. J.; Klene, M.; Knox, J. E.; Cross, J. B.; Bakken, V.; Adamo, C.; Jaramillo, J.; Gomperts, R.; Stratmann, R. E.; Yazyev, O.; Austin, A. J.; Cammi, R.; Pomelli, C.; Ochterski, J. W.; Martin, R. L.; Morokuma, K.; Zakrzewski, V. G.; Voth, G. A.; Salvador, P.; Dannenberg, J. J.; Dapprich, S.; Daniels, A. D.; Farkas, Ö.; Foresman, J. B.; Ortiz, J. V.; Cioslowski, J.; Fox, D. J. Gaussian, Inc., Wallingford CT, 2009. 
References:

(1) Knight, A. S.; Zhou, E. Y.; Pelton, J. G.; Francis, M. B. Selective chromium(VI) Ligands Identified Using Combinatorial Peptoid Libraries. J. Am. Chem. Soc. 2013, 135 (46), 17488.

(2) Maltese, M. Reductive Demercuration in Deprotection of Trityl Thioethers, Trityl Amines, and Trityl Ethers. J. Org. Chem. 2001, 66 (23), 7615.

(3) Zuckermann, R. N.; Kerr, J. M.; Kent, S. B. H.; Moos, W. H. Efficient Method for the Preparation of Peptoids [oligo(N-Substituted Glycines)] by Submonomer Solid-Phase Synthesis. J. Am. Chem. Soc. 1992, 114 (26), 10646.

(4) Kremleva, A.; Krüger, S.; Rösch, N. Role of Aliphatic and Phenolic Hydroxyl Groups in Uranyl Complexation by Humic Substances. Inorganica Chim. Acta 2009, 362 (8), 2542. 